\title{
Beam Based Nonlinear Corrections in Storage Rings: Review and Applications for the LHC Commissioning
}

\author{
O. Brüning, \\ CERN, Geneva, Switzerland
}

\begin{abstract}
After summarising the required correction circuits in the LHC we will give a short summary of potential tools for measuring non-linear resonances and error distributions in a storage ring. The aim is to identify the limits and strength for each method and to identify how they can be used for adjusting the different correction circuits in the LHC.
\end{abstract}

\section{INTRODUCTION}

The performance of the Large Hadron Collider (LHC) depends to large extend on a proper setting of the non-linear correction circuits. The LHC machine features non-linear correction elements in each of its eight arcs and on each side of its four experimental insertions. The LHC arcs have two different types of correction circuits:

- spool piece corrector magnets which are mounted directly to the end of the main dipole magnets are foreseen for the $b_{3}, b_{4}$ and $b_{5}$ multipole errors. (We use the European notation and $b_{3}$ denotes an upright sextupole error.)

- lattice corrector circuits which are mounted to the main arc quadrupole magnets. Lattice corrector circuits are foreseen for the $a_{2}, b_{2}, a_{3}, b_{3}$ and $a_{4}$ field components. Each arc has four $b_{3}$ circuits per beam for correcting the natural and second order chromaticity of the machine and one $a_{3}$ circuit for correcting the chromatic coupling in the machine. Two $b_{4}$ circuits (OD and $\mathrm{OF}$ ) can generate transverse Landau damping at top energy.

In total there are 9 non-linear circuits per arc yielding a total of 72 circuits for the eight arcs.

Moreover, five non-linear correction coils compensate the triplet field errors on each side of the experimental insertions. Correction coils are foreseen for the $a_{3}, b_{3}, a_{4}, b_{4}$ and $b_{6}$ multipole errors. The LHC has four experimental insertions yielding a total number of 40 correction circuits. Together with the arc correction circuits the LHC has a total of 112 non-linear correction circuits for the whole LHC machine.

Each LHC magnet will be measured cold before installation in the tunnel and the measured field errors will be used to preset the non-linear correction circuits of the machine. During the LHC commissioning the polarity and proper connection of each circuit has to be verified and each element of the correction circuits must be tested against a short circuit. Because the correction circuits are superconducting magnets, a magnet short can only be detected during warm magnet tests or via beam based measurements in the cold state of the machine.

Moreover, the powering of the correction circuits must be readjusted during the machine operation. The persistent current errors in the super-conducting magnets of the LHC change by approximately $30 \%$ over a time period of approximately 30 minutes. For example, the decay of the persistent current $b_{3}$ error of the main dipole fields is approximately $b_{3}=-3.6 \cdot 10^{-4}$. One unit of $b_{3}$ corresponds to approximately 45 units of chromaticity and the total change in chromaticity due to the persistent current decay at $450 \mathrm{GeV}$ is

$$
\Delta Q^{\prime}(\text { injection })=160,
$$

which is approximately 100 times larger than the maximum permissible chromaticity change during the machine operation. At $7 \mathrm{TeV}$ the persistent current decay amounts to 0.06 units and the change in chromaticity due to the persistent current decay becomes

$$
\Delta Q^{\prime}(\text { injection })=3
$$

which is just on the border of requiring correction. At the beginning of the ramp the persistent current errors change abruptly back to their initial values. The speed of this 'snap back' effect depends on the speed of the current change at the beginning of the ramp and occurs during the first $50 \mathrm{~A}$ of the ramp. During the ramp the multipole errors change due to ramp induced current perturbations. The field amplitude of these errors is proportional to the ramp speed. The goal for the LHC operation is to assure a $b_{3}$ correction with an error of less then $0.5 \%$ at all stages of the machine operation. All other non-linear correction circuits require a correction within $80 \%$ to $90 \%$ of the magnetic field errors. One of the key challenges for the LHC commissioning will be to find proper beam observables for the verification of the correction circuit settings at each stage of the machine operation.

\section{NON-DESTRUCTIVE MEASUREMENT TECHNIQUES}

\subsection{Measurement of the field error feed down with local orbit bumps}

A transverse orbit displacement inside a multipole field imperfection generates feed down errors of [1]

$\left(b_{n-k}+i a_{n-k}\right)=\frac{(n-1) ! \cdot\left(b_{n}+i a_{n}\right)}{(n-k-1) ! \cdot k !} \cdot \frac{(\triangle x+i \triangle y)^{k}}{R_{r}^{k}}$ 
where $n$ is the order of the original error, $\triangle x$ and $\triangle y$ the horizontal and vertical orbit displacements inside the original multipole error, $k$ the order of the feed down and $R_{r}$ the reference radius for the field error expansion $\left(R_{r}=17 \mathrm{~mm}\right.$ for the LHC magnets). For example, a horizontal orbit displacement inside a sextupole field imperfection generates a quadrupole error

$$
b_{2}=2 \cdot \frac{\triangle x}{R_{r}} \cdot b_{3} .
$$

The normalised quadrupole gradient is given by

$$
k=\frac{b_{2}}{R_{r} \cdot \rho},
$$

where $\rho$ is the bending radius inside the dipole magnets $(\rho \approx 2800 \mathrm{~m})$. The quadrupole feed down error generates a total tune change of

$$
\triangle Q=\frac{1}{4 \pi} \cdot \int_{\text {orbitbump }} \beta(s) \cdot k(s) d s
$$

where the integral extends over the length of the orbit bump. Thus, using local orbit bumps and measuring the total tune change in the machine provides direct information on the local sextupole field error in the machine. For example, let us assume a $20 \%$ mispowering of the sextupole spool piece circuit in one of the eight LHC arcs and a global correction of the machine chromaticity via the lattice sextupole circuits (distributed over all eight arcs). All other sextupole spool piece circuits are correctly powered. We can measure the mispowering by generating a set of seven $\pi$-bumps with a peak amplitude of $3 \mathrm{~mm}$ and moving the bumps from arc to arc in the LHC machine. When the orbit bumps are in the arc with the mispowered spool piece circuit one can observe a total tune change of $\triangle Q=0.01$. If the $\pi$-bumps are generated in any of the other arcs the procedure yields a tune change of less then $\triangle Q<0.001$.

Alternatively, one can use a dispersion bump that extents over a whole arc (systematic dipole error over one arc of the machine) for verifying the spool piece settings in the different arcs of the LHC machine.

An orbit bump of $3 \mathrm{~mm}$ significantly reduces the mechanical aperture of the machine and the procedure can be further optimised by using time varying instead of static orbit bumps. If the amplitude of the orbit bumps oscillates in time the resulting tune modulation can be measured via a phase locked loop (PLL). PLL based tune measurements allow a detection of tune variations with an amplitude of $\triangle Q=10^{-5}$. Generating a tune modulation which is more then one order of magnitude larger than the above PLL resolution $\left(\triangle Q=3 \cdot 10^{-4}\right)$ requires for the above spool piece error scenario an orbit excursion of only $\triangle x=0.1 \mathrm{~mm}$. In the case of the LHC orbit corrector magnets the above orbit modulation can be generated at a frequency of $1 \mathrm{~Hz}$ at injection energy. Faster modulation frequencies are only possible if more powerful power converters are available.

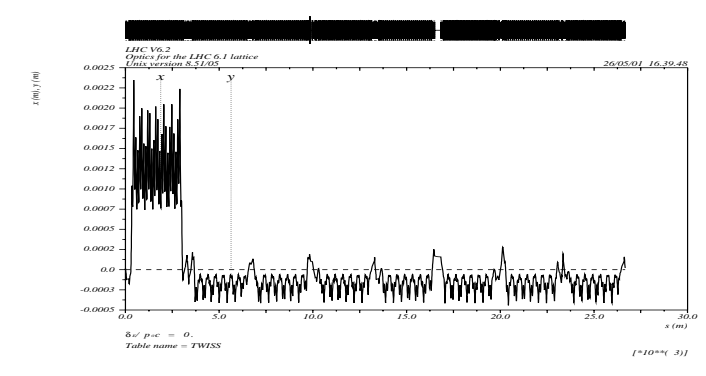

Figure 1: The horizontal orbit in the LHC for a dispersion bump in arc12.

At this point it is worthwhile noting that the above procedure can not measure the total sextupole error in the machine (and thus the machine chromaticity). The RF system of a synchrotron keeps the total orbit length constant and any horizontal orbit displacement inside the bending magnets must be compensated by an equivalent displacement with opposite sign at another location of the machine. For example, Fig. 1 shows the total horizontal orbit for a dispersion bump in one of the eight LHC arcs. One clearly recognises how the orbit in the whole machine is changed in order to compensate for the local orbit bumps in the first LHC arc. This limitation does not exist for orbit bumps in dispersion free areas ( $\rightarrow$ vertical orbit bumps in the arcs or horizontal/vertical orbit bumps in the straight sections). For example, a vertical orbit offset in a sextupole field imperfection changes the machine coupling and a measurement of the total machine coupling as a function of local vertical orbit bumps can provide information on the average sextupole error in the machine. However, in practice it might be difficult to measure the machine coupling in a non-destructive manner.

Local orbit bumps are particularly interesting for an adjustment of the corrector magnets inside a dispersion free area, e.g. the focusing quadrupoles left and right from the experimental areas. In this case local orbit bumps for the feed down measurement change the orbit in the rest of the machine insignificantly. This method has been successfully applied to the adjustment of the insertion region corrector circuits in the RHIC accelerator and might be applicable for the $a_{3}, b_{3}$ and $a_{4}$ LHC triplet corrector circuits [2].

In summary one can note that orbit bump measurements are a perfect tool for detecting short circuits or polarity errors in the correction magnets during the machine commissioning. Provided the machine control and beam instrumentation system allow the generation and detection of time varying orbit bumps and tune modulations, the method can also provide non-destructive online measurements of the correction circuit powering during the nominal machine operation.

\subsection{Reconstructing Hamiltonian Coefficients}

An even more ambitious application of orbit bumps is the full reconstruction of the coefficients in the Hamilto- 
nian that describes the particle motion in a storage ring [3]. The particle motion in a linear system perturbed by point like sextupole kicks distributed along the azimuth of the storage ring is described by a third order Hamiltonian with 20 monomials. The goal of the 'orbit wobbling' method described in [3] is to determine the 20 unknown coefficients of this Hamiltonian via orbit oscillations. Modulating the closed orbit in the machine with four different excitation frequencies using four different orbit corrector magnets (two horizontal and two vertical corrector magnets) the third order Hamiltonian system mixes the four excitation frequencies to 17 different response frequencies. Observing these 17 different response frequencies at four different BPMs (2 horizontal and 2 vertical BPMs) yields a total of 68 different observables which allow a full reconstruction of the Hamiltonian system. The simulations in [3] demonstrate that the above procedure is robust against BPM errors but does not address the perturbation from higher order multipole errors in the machine. The above procedure has been tried out at the COSY cooler synchrotron [4]. Unfortunately, it was difficult to interpret the measured data and more detailed studies are required before the method can be applied to the LHC operation.

\section{CHROMATICITY MEASUREMENT TECHNIQUES}

The third order Taylor expansion of the machine tune in terms of the particle momentum error can be written as

$$
Q=Q_{0}+Q^{\prime} \cdot \frac{\delta p}{p_{0}}+\frac{1}{2} \cdot Q^{\prime \prime} \cdot\left(\frac{\delta p}{p_{0}}\right)^{2}+\frac{1}{6} \cdot Q^{\prime \prime \prime} \cdot\left(\frac{\delta p}{p_{0}}\right)^{3}
$$

The $b_{3}$ error changes the linear machine chromaticity, the $a_{3}$ and $b_{4}$ errors and the high $\beta$-functions inside the triplet quadrupole magnets generate a second order chromaticity $\left(Q^{\prime \prime}\right)$ and the $b_{5}$ error a third order chromaticity $\left(Q^{\prime \prime \prime}\right)$.

Measuring the linear machine chromaticity provides information on the global sextupole error in the machine.

The second order chromaticity generated by the $a_{3}$ field components depends on the separation of the horizontal and vertical tunes. The $a_{3}$ error leads to momentum dependent coupling coefficient given by [5]

$$
c_{-}=\int \frac{a_{3} \cdot D_{x} \cdot \sqrt{\beta_{x} \beta_{y}}}{\pi} \cdot e^{i\left(\mu_{x}-\mu_{y}\right)} \cdot \frac{\delta p}{p_{0}} d s
$$

where $D_{x}$ is the horizontal dispersion in the machine and $\mu_{x}$ and $\mu_{y}$ the horizontal and vertical phase advance respectively. The coupling coefficient leads to a tune separation of

$$
\triangle Q=\sqrt{\left(Q_{I}-Q_{I I}\right)^{2}+\left|c_{-}\right|^{2} \cdot\left(\frac{\delta p}{p_{0}}\right)^{2}} .
$$

Expanding Equation (9) to second order in $\left(\delta p / p_{0}\right)$ yields

$$
Q_{I, I I}=Q_{x, y} \pm \frac{\left|\int a_{3} \sqrt{\beta_{x} \beta_{y}} e^{i\left(\mu_{x}-\mu_{y}\right)} D_{x} d s\right|^{2}}{4 \pi^{2} \triangle Q_{0}}\left(\frac{\delta p}{p_{0}}\right)^{2}
$$

and one obtains a contribution to the second order chromaticity which depends on the separation of the horizontal and vertical tune of the machine. The measurement procedure for an $a_{3}$ correction is as follows: one varies the beam momentum via RF frequency adjustments with a tune feedback on and estimates the tune change (and thus the chromaticity) from the tune adjustments of the feed-back loop. One repeats the measurement for different tune separations. If the measurement yields different chromaticity values for different tune separations it indicates an uncorrected $a_{3}$ error.

The $b_{5}$ error generates a third order chromaticity of [2]

$$
Q^{\prime \prime \prime}=\frac{6}{\pi R_{r}^{4}} \int \frac{b_{5} \cdot \beta \cdot D_{x}}{\rho} d s .
$$

The current error table of the LHC dipole magnets has a total uncorrected systematic decapole error of $b_{5} \approx 10^{-4}$ at injection energy. Assuming a $20 \%$ unbalance of the spool piece circuits this generates a third order chromaticity of

$$
Q^{\prime \prime \prime} \approx 0.8 \cdot 10^{6} \rightarrow \triangle Q\left(\delta p / p_{0}=2 \cdot 10^{-3}\right) \approx 10^{-3}
$$

which is large enough to be detected in a chromaticity measurement. The main challenge of this measurement procedure is the reduced aperture due to the momentum shift. The peak horizontal dispersion inside the LHC arcs is $D_{x}=2.05$ meter and the above momentum shift of $\delta p / p_{0}=2 \cdot 10^{-3}$ generates an additional orbit displacement of $4 \mathrm{~mm}$ in the arcs.

In summary, it can be noted that linear and non-linear chromaticity measurements provide information on the global correction of the most important field errors in the LHC magnets. Important questions for the applicability of the measurements in the nominal machine operation is the speed at which the chromaticity can be measured, the maximum momentum deviation for the chromaticity measurement and whether the measurement is destructive for the stored beam.

Apart from the classical chromaticity measurement via RF frequency shifts, there are currently several new techniques for the chromaticity measurement under study[6][9]. Table 1 summarises the pro and cons for the different measurement options. There is not one single chromaticity

\begin{tabular}{|c|c|c|}
\hline Technique & Limit & Strength \\
\hline RF frequency shift & slow & large $\delta p / p_{0}$ \\
\hline off momentum ramps & several ramps & large $\delta p / p_{0}$ \\
\hline head tail oscillations & beam blow up & fast \\
\hline RF phase modulation & small $\delta p / p_{0}$ & fast \\
\hline RF $\pi$-bump & RF phase loop & fast \\
\hline
\end{tabular}

Table 1: Potential measurements techniques for the chromaticity measurement.

measurement technique which covers all the applications for the LHC operation. The first two methods can generate a large $\delta p / p_{0}$ and, thus, allow the measurement of the nonlinear chromaticity. The third method, the head tail measurement, is the only fast chromaticity measurement that 
has been demonstrated to work in existing storage rings. Unfortunately, it requires large beam excitations and is destructive. The LHC requires at least measurements with the first and third method. The last two measurement options are new proposals for fast, non-destructive measurements of the linear chromaticity. However, they have not yet been fully tested in existing storage rings and more studies are required before they can be applied in the LHC operation.

\section{DESTRUCTIVE MEASUREMENT TECHNIQUES}

Kicking the beam in the storage ring allows a Fourier analysis of the particle motion and opens a variety of possibilities for analysing the non-linear particle motion. Unfortunately, such a beam excitation results in an emittance blow-up in proton storage rings (no radiation damping) and thus requires dedicated beams for the measurements. In the following we will discuss three different measurement methods.

- tune measurements as a function of oscillation amplitude

- evaluation of the resonance driving terms from a complex Fourier analysis of the particle motion

- analysis of the frequency map between initial conditions in the transverse plane and the tune diagram.

\subsection{Tune Measurements as a Function of Oscil- lation Amplitude}

The LHC kicker magnets are designed to generate a peak oscillation amplitude of $8 \sigma$ at top energy ( $7 \mathrm{TeV})$. The kick amplitude can be modulated over one bunch train (ca. 80 bunches) so that each bunch obtains a different oscillation amplitude. Measuring the tune in the machine for each bunch provides a fast measurement of the machine anharmonicity. The anharmonicity can be either directly generated by octupole field errors $\left(b_{4}\right)$ or as a second order effect from sextupole field components $\left(b_{3}\right)$. In both cases the anharmonicity measurement can be used as an observable for the global adjustment of the $b_{3}$ and $b_{4}$ spool piece circuits.

\subsection{Measuring Resonance Driving Terms via complex Fourier Transforms}

Measuring simultaneously the readings at two BPMs which are $90^{\circ}$ apart in $\beta$-tron phase provides information on the particle position and the particle momentum at the first BPM. Describing the time series of the position and momentum readings in a quasi-periodic approximation

$$
x(N)+i \cdot p_{x}(N)=\sum_{j} a_{j} \cdot e^{i\left[2 \pi\left(m_{j} Q_{x}+n_{j} Q_{y}\right) \cdot N+\phi_{j}\right]}
$$

allows the evaluation of the individual resonance driving amplitudes in Equation (13). Identifying the proper reso- nance coefficients in Equation (13) requires a precise measurement of the horizontal and vertical particle tunes. Unfortunately, the BPM response typically diminishes within a few thousand turns due to the non-linearity of the particle motion and the tune measurement can rely only on a few hundred data points. The frequency analysis only became possible with the development of superior tools for the Fourier analysis. Using an analytical interpolation of the data points and a proper windowing of the data sets the work in [10], [11] and [12] showed that the tune can be measured with an accuracy of $1 / N^{4}$ for tracking data and $1 / N^{2}$ in the presence of noise in the BPM readings, where $N$ is the number of data points. (A normal FFT provides a tune estimate with an accuracy of $1 / N$.)

Comparing the spectral lines in the Fourier spectrum with the central tune lines provides information on the amplitude of the resonance driving terms [13][14]. Measuring the spectral line amplitudes for different oscillation (kick) amplitudes provides information on the amplitude dependence of the resonance driving terms and measuring the spectral line amplitudes for different BPMs around the machine provides information on the azimuthal distribution of the field errors which generate the resonance driving terms. The method thus provides the possibility of a full reconstruction of the error multipole distribution in the storage ring. First measurements in the SPS [14] are encouraging and provide good results for an SPS machine with localised sextupole perturbations. However, the measurement of higher order multipoles is quite challenging and requires further studies on the effect of BPM non-linearities and BPM resolution.

\subsection{Frequency Map Analysis}

Generating a quasi-periodic approximation of the particle motion for various initial conditions and retaining only the frequency vector $\left(Q_{x}, Q_{y}\right)$ which parametrises the KAM tori in the stable regions of a non-degenerate Hamiltonian system [15] provides a frequency map (FM) for the Hamiltonian system. For example, for the Hamiltonian system of an accelerator one can reduce the four-dimensional transverse phase space by keeping all the position variables constant and varying only the horizontal and vertical initial momenta (kick amplitudes) which produces a map

$$
\mathcal{F}_{\tau}: \begin{gathered}
R^{2} \\
\left.\left(I_{x}, I_{y}\right)\right|_{p_{x}, p_{y}=0},
\end{gathered} \quad \begin{gathered}
R^{2} \\
\left(Q_{x}, Q_{y}\right)
\end{gathered} .
$$

The dynamics of the particle motion inside the storage ring can be analysed by studying the regularity of this map and the above method has been successfully used in the ALS operation [16].

Figure 2 shows the FM for tracking data based on the LHC optics version 5. One clearly recognises the deteriorating effect of the resonance lines $(7,0)$ and $(3,7)$ on the regularity of the points in the FM. However, deteriorating effects of the above resonances only show up at very large oscillation amplitudes $(\triangle x>10 \sigma)$ and it is not clear if this method can be applied to the LHC operation. 


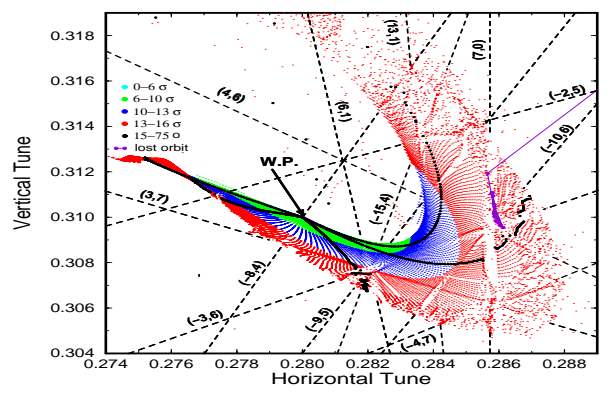

Figure 2: Frequency map for the LHC optics version 5.

\section{NEW TOOLS}

Exciting the beam oscillations with a sinusoidal modulation of a dipole field instead of an individual dipole kick can produce a long lasting signal of the beam oscillations (no decoherence of the signal due to filamentation) without increasing the beam emittance [17]. The method requires dipole field oscillation close to the betatron frequency. (but outside the frequency spectrum of the beam) which can be adiabatically turned on and off. A dipole which satisfies the above criteria is called an AC-dipole. If the ACdipole frequency lies inside the beam spectrum or if the excitation is turned on or off non-adiabatically the measurement will blow up the beam emittance. First tests have been performed at the AGS in BNL [18] using an air coil dipole magnet which was originally proposed spin manipulations and at the CERN SPS using the transverse damper kicker magnets [19]. Both measurements could successfully generate beam excitations without measurable emittance blowup. The AC-dipole excitations provide an enormous potential for future diagnostics. For example, combining the AC-dipole excitation with the methods presented in Section 4 could open the possibility of having online measurements of the resonance driving terms in the machine without deteriorating the beam quality. However, further experimental studies at existing storage rings are required before this method can be applied for machine diagnostics in a routine machine operation.

\section{OTHER TOOLS FOR OPERATION OPTIMISATION}

Measuring and analysing the loss rates at the primary collimator jaws provides information on the frequency spectrum of the particle motion and the local diffusion coefficients at the positions of the collimator jaws [20]. This information can be used for verifying the beneficial effect of any change in the correction circuit powering and thus, provides an important tool for cross-checking the results of readjustments in the correction circuit powering. Provided the number of relevant correction circuits is limited, this method might even be used for an empirical adjustment of the correction circuit powering. However, considering the large number of correction circuits in the LHC this last application seems to be less likely.

\section{SUMMARY}

The LHC operation requires a global correction of the sextupole errors $\left(b_{3}\right)$ with a maximum error of $0.3 \%$ and a local correction with a maximum error of $10 \%$. All other arc correction circuits require corrections in the $10 \%$ to $20 \%$ accuracy. Existing measurement techniques provide potential information on the global and local correction of almost all the non-linear correction circuits in the LHC:

- Sextupole field errors $\left(b_{3}\right)$ can be detected by measuring the machine tune versus horizontal orbit bump amplitudes, measuring the linear chromaticity and Fourier analysing the time series of BPM data ( $\rightarrow$ resonance driving term analysis)

- Skew sextupole field errors can be detected by measuring the second order chromaticity and the machine tune versus vertical orbit bump amplitudes

- Octupole field errors $\left(b_{4}\right)$ can be detected by measuring the machine tune as a function of the oscillation amplitudes, measuring the second order chromaticity and Fourier analysing the time series of BPM data (measuring the $(2,-2)$ resonance)

- Skew octupole field errors can be detected by Fourier analysing the time series of BPM data (measuring the $(1,-1)$ resonance) provided the BPM non-linearity is not too large

- Decapole field errors $\left(b_{5}\right)$ can be detected by measuring the third order chromaticity

A beam-based adjustment of the dodecapole $\left(b_{6}\right)$ correction circuits in the triplet quadrupole magnets seems to be less obvious and requires further studies [2].

\section{REFERENCES}

[1] R. Bartolini and W. Scandale, LHC-MMS 97-12, 1997

[2] J.-P. Koutchouck et al, TPPH013, this conference.

[3] V. Ziemann, Particle Accelerators 54/55, pg [419]/173 1996

[4] P. Leunissen, Ph.D. thesis, T. Universiteit Eindhoven 1997.

[5] S. Fartoukh, LHC Project Report 278, 1998.

[6] S. Herb et al, Harz Seminar Proceedings, Badlauterberg 1992

[7] D. Cocq et al, $8^{\text {th }}$ Workshop on Beam Instr., Stanford, 1998

[8] T. Linnecar and O. Brüning, ongoing studies at CERN (SPS)

[9] J.P. Koutchouck and J. Tückmantel, ongoing studies

[10] J. Laskar et al, Astron. Astroph. 198, 341, 1988

[11] E. Asseo et al, 4. Europ. Signal Processing Conf., 1988

[12] R. Bartolini et al, Part. Accel. 52, 1996

[13] R. Bartolini et al, PAC1999, New York 1999

[14] F. Schmidt et al, this conference WOPB004

[15] Y. Papaphilippou, PAC1999, New York 1999

[16] D. Robin et al, EPAC2000, Vienna 2000

[17] S. Peggs, PAC1999, New York 1999

[18] M. Bai et al, Phys. Rev. E56, 1997

[19] O. Berrig et al, DIPAC2001, Grenoble 2001

[20] M. Seidel, DESY 94-103, 1994 